\title{
Stirring the embers: mesenteric panniculitis provoked by colonoscopy
}

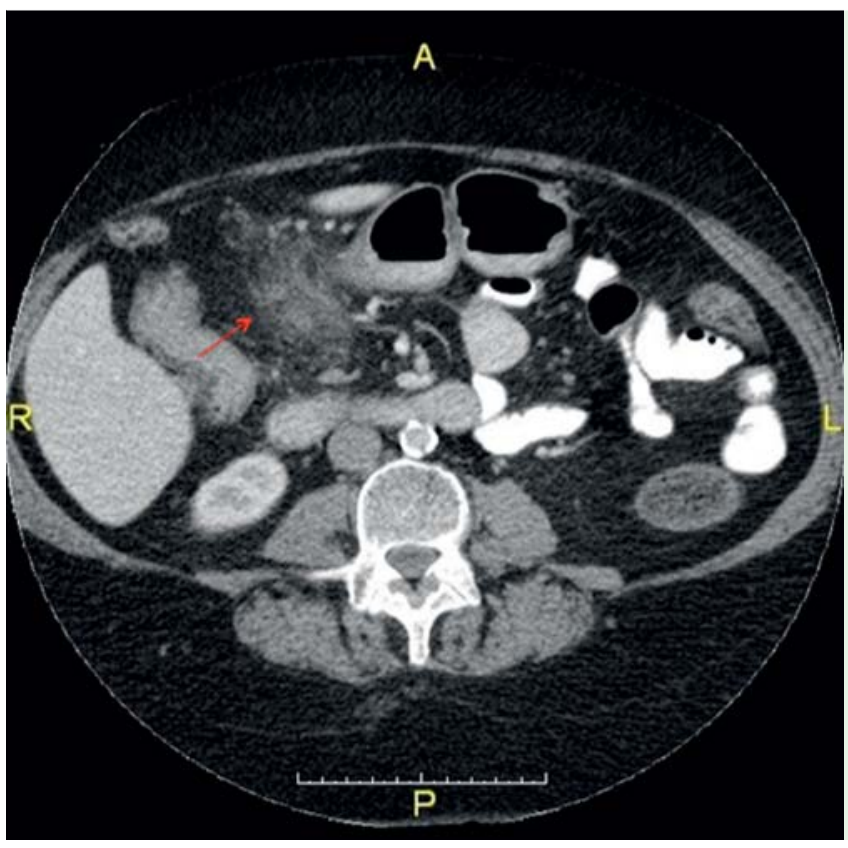

Fig. 1 Computed tomography of the abdomen and pelvis in a 73-year-old woman with fever and abdominal pain that developed immediately after colonoscopy. The scan reveals an infiltrative pattern (arrow) and slightly enlarged lymph nodes within the root of the mesentery. A, anterior; $\mathrm{P}$, posterior; R, right; L, left.

Severe abdominal pain following colonoscopy usually reflects retained gas [1], but on rare occasions, it may reflect more serious problems. Here, we report a rare cause.

A 73-year-old woman presented for routine ambulatory colonoscopy. A few months before the procedure, she had begun to experience unexplained intermittent night sweats, arthralgia, and vague abdominal pain. The results of blood testing and computed tomography of the abdomen and pelvis were unrevealing.

On the day of the colonoscopy, the patient felt well. A 2-cm polyp was resected from the cecum without incident. Immediately after the procedure, abdominal pain and fever developed. Computed tomography with oral contrast and with and without intravenous contrast, performed the next day ( Fig. 1), showed colonic diverticulosis and new, hazy infiltration within the mesenteric fat superior and posterior to the proximal transverse colon. The area of inflammation was not contiguous with the site of the polypectomy, which appeared unremarkable. A diagnosis of mesenteric panniculitis was made.
On hospital admission, the C-reactive protein level was $273 \mathrm{mg} / \mathrm{L}$ (normal 0 - $5 \mathrm{mg} / \mathrm{L}$ ) and the white blood cell count was $20.9 \times$ $10^{3} / \mu \mathrm{L}$ (normal $4.5-11.0 \times 10^{3} / \mu \mathrm{L}$ ). Intravenous hydrocortisone sodium succinate was begun. Within 24 hours, the patient became afebrile with reduced abdominal pain. The inflammatory markers decreased rapidly.

Mesenteric panniculitis is a rare fibro-inflammatory disease [2-4] whose etiology is unknown. The diagnosis is established by the presence of classic radiologic findings [3]. One previous case of mesenteric panniculitis occurring after colonoscopy has been reported [4]. In that case, complex sigmoid polypectomy procedures resulted in a transmural inflammatory reaction at the polypectomy sites. In our case, the mesenteric inflammation was remote from the polypectomy site, did not involve the colonic wall, and resolved with the intravenous administration of corticosteroids. We speculate that the patient had "smoldering" panniculitis, and that this inflammation was provoked by the instrumentation.
Competing interests: None

Kathryn B. Friedman ${ }^{1}$, Burton Cohen², Louis R. DePalo ${ }^{3}$, James Aisenberg ${ }^{4}$

${ }^{1}$ Department of Medicine, Icahn School of Medicine at Mount Sinai, New York, New York, USA

2 Department of Radiology, Icahn School of Medicine at Mount Sinai, New York, New York, USA

${ }^{3}$ Department of Medicine, Division of Pulmonary and Critical Care Medicine, Icahn School of Medicine at Mount Sinai, New York, New York, USA

${ }^{4}$ Department of Medicine, Division of Gastroenterology, Icahn School of Medicine at Mount Sinai, New York, New York, USA

\section{References}

1 Geyer M, Guller U, Beglinger C. Carbon dioxide insufflation in routine colonoscopy is safe and more comfortable: results of a randomized controlled double-blinded trial. Diagn Ther Endosc 2011; 2011: 378906

2 Van Lingen CP, Zeebregts CJ, Gerritsen JJ et al. Mesenteric panniculitis presenting as colitis. ANZ J Surg 2004; 74: 176

3 Daskalogiannaki M, Vouloudaki A, Prassopoulos $P$ et al. CT evaluation of mesenteric panniculitis: prevalence and associated diseases. AJR Am J Roentgenol 2000; 174: 427 431

4 Lee KJ, Ehrenpreis ED, Greenberg $J$ et al. Mesenteric panniculitis following colonoscopy, polypectomy, and epinephrine injection. Endoscopy 2010; 42: E44-E45

\section{Bibliography}

Dol http://dx.doi.org/

10.1055/s-0034-1392864

Endoscopy 2015; 47: E470

(c) Georg Thieme Verlag KG

Stuttgart · New York

ISSN 0013-726X

\section{Corresponding author}

\section{Kathryn B. Friedman}

311 East 79th Street

Suite 2A

New York, NY 10075

USA

Fax: +1-212-996-6677

Kathryn.friedman@icahn.mssm.edu 